\title{
The electrical conductivity behavior of an organic insulator caused by presence of dead ends under high electric field
}

\author{
Amina BENALLOU* ${ }^{* 1}$, Baghdad HADRI ${ }^{1}$, Juan MARTINEZ-VEGA ${ }^{2}$ \\ ${ }^{1}$ Laboratoire d'électromagnétisme et optique guidée LEGO, Université Abdelhamid Ibn Badis, \\ Mostaganem, Mostaganem, Algeria \\ ${ }^{2}$ Laboratoire d'électromagnétisme et optique guidée, Université de Toulouse; UPS, LAPLACE \\ F-31062, CNRS; Toulouse, France.
}

*Corresponding author: amina.benallou@gmail.com

Keywords: Polymers, Electrical Conductivity, Organic Insulator, Percolation, Dead End

\begin{abstract}
In this paper, an analytical model based on the percolation theory and a numerical model based on Monte Carlo method was developed and used to study the electrical conductivity behavior of an organic insulator under high electric field. The percolation model considers that the real network of organic insulator is consisted of the traps randomly distributed and connected to each other by conductance $\mathrm{G}_{\mathrm{ij}}$. The phenomenon of charges trapping and detrapping depends strongly on certain parameters such as the intensity of the applied electrical field, the dead ends length of traps, and the random distribution of the energies of traps constituting the dead end. Using gaussian energy distribution negative electrical conductivity law as exponential function of the electric field was found. The simulation results were compared with an analytical model, they are in agreement, proving the accuracy of this model.
\end{abstract}

\section{Introduction}

The transport phenomenon in the organic insulators (polymers) was treated by using the percolation theory $[1,2]$. When a percolation network such as the organic insulator reaches percolation threshold that is the critical concentration of traps by which a cluster of infinite traps appears. This cluster consists of finite set of clusters of traps connected to each other them and connecting the two electrodes. This infinite cluster (cluster percolating) contains additional finite clusters of particular traps that are connected to it only on one side .They are called dead ends.

The percolation model could be apply in order to determine the electrical conductivity behavior as the function of electric field. The dead ends of traps are considered to be not participate to the process of conduction in the organic materials when a weak electric field is applied $[3,4]$. In a high electric field, the dead end effect is often neglected during the electrical conduction modeling. In this paper, it will be developed an analytical model based on the percolation theory. It could be explain the fluctuations caused by the dead ends of traps on the behavior of electrical conductivity, in high electric field region. This model will be validated by a numerical simulation.

\section{Percolation approach}

The charge carriers are trapped by the dead ends during a rather long time of stay. However, when the organic insulator material is subjected to the high electrical field effect, trapping time increases until some localized charge carriers are detrapped and trap again on other traps belonging to dead end and so on. This phenomenon of trapping and detrapping depends strongly on certain parameters such as the intensity of the applied electrical field, the length of the dead ends of traps, and the random distribution of the energies of traps constituting the dead end [5]. 


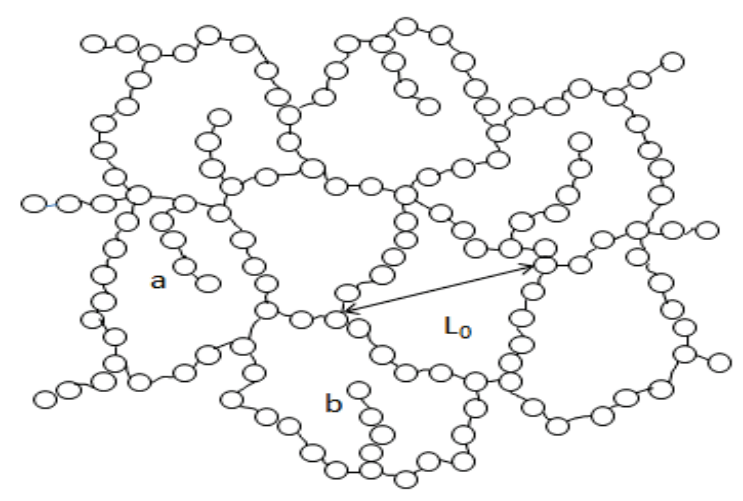

Fig.1. Schematic representation of a portion of infinite cluster constituted of a set of finite clusters of length $L_{0}$ containing dead ends (a) and (b).

After calculated the total number of finite clusters trap in a dead end in order to determine the electrical conductivity versus the electric field for Gaussian distribution.

The probability of finding a dead end of traps with length $\mathrm{x}_{0}$ for Gaussian distribution is determined as follow:

$$
\mathrm{p}\left(\mathrm{x}_{0}, \delta\right) \cong \exp \left(-\frac{\sqrt{\pi} \mathrm{x}_{0} \delta}{\mathrm{L}_{0}}\right)
$$

Where $\mathrm{p}$ is the probability of finding a dead end of traps with length $\mathrm{x}_{0}$, $\mathrm{L}_{0}$ : correlation length

$$
\delta=\frac{\mathrm{eEx}_{0}}{\mathrm{kT}} \text { Dimensionless electric field }
$$

Electron average residence time in the dead end is determined as

$$
\prec t \succ \cong \mathrm{t}_{0} \exp \left[\frac{\mathrm{eEL}}{4 \sqrt{\pi} \mathrm{kT}}-\frac{\mathrm{eEL}}{8 \sqrt{\pi} \mathrm{kT}}\right]
$$

$\mathrm{t}_{0}$ is residence time without electric field $\mathrm{E}, \mathrm{k}$ is Boltzmann constant and $\mathrm{T}$ is temperature Electrical conductivity is determined as

$$
\sigma(\mathrm{E}, \mathrm{W}) \cong \sigma_{0} \exp \left[-\frac{\mathrm{eEL} \mathrm{L}_{0}}{8 \sqrt{\pi} \mathrm{kT}}\right]
$$

Where $\sigma_{0}$ Electrical conductivity without electric field.

\section{Simulation}

Previously it has been developed and showed that, the dead ends of traps effect on the behavior of the electrical conductivity in a high electrical field. When the carriers of charges were trapping by the dead end of traps, the behavior of the electrical conductivity were decaying exponentially. In other words the dead ends of traps decrease a part of the electrical conductivity according to the distribution of the energies of the traps. The simulation is to demonstrate and validate the effect of fluctuations in dead ends of traps on the electrical conductivity.

We note that the electrical conductivity curve of the simulated organic insulating shows fluctuations for values of electric field. To check if the dead end of traps affects the electrical conductivity, it should be deleting to see if the compensation of the fluctuation will take place. We have cutting some dead ends. Our simulated results shown in figure 2 that. The simulation results show firstly that electrical conductivity curve (black) $\sigma_{1}=\mathrm{f}(\mathrm{E})$ is completely different to $\sigma_{2}=\mathrm{f}(\mathrm{E})$ 
electrical conductivity when a few dead end are cutting (red). So the dead ends are responsible for the observed fluctuations on the electrical conductivity curve.

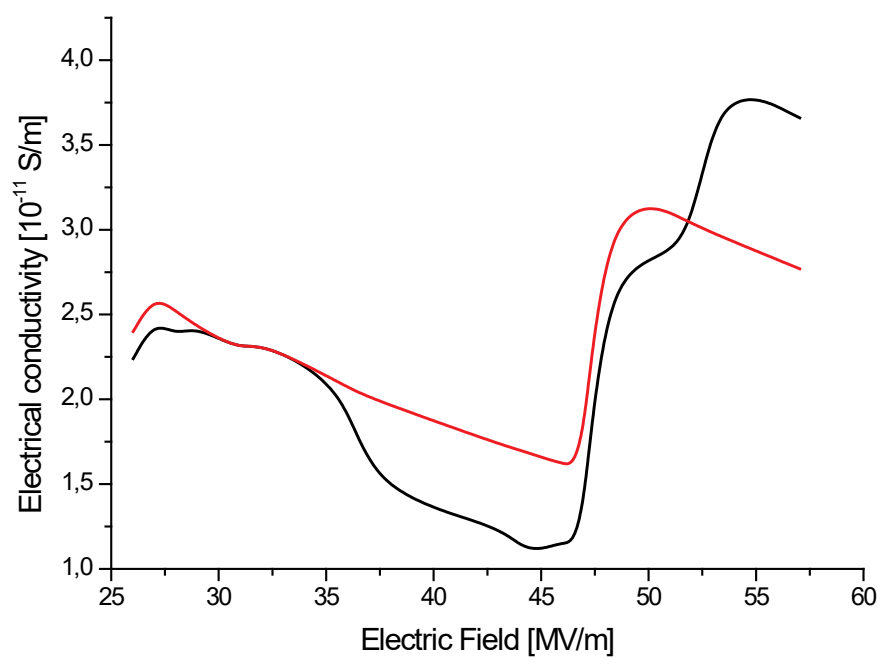

Fig.2. Comparison between electrical conductivity versus electric field. $\sigma_{1}=f(E)$ global electrical conductivity (black). $\sigma_{2}=f(E)$ electrical conductivity when a few dead end are cutting ( red).

\section{Conclusion}

From the research that has been carried out, we can conclude that the electrical conductivity as function of electric field in an organic insulator was studied by using percolation approach. It was shown that the electrical conductivity due to dead ends of traps were decaying. By employing the simple Monte Carlo algorithm in order to simulate the electrical conductivity behavior versus the electric field with the presence of dead ends of traps, the numerical results were showed fluctuations in electrical conductivity curve. These fluctuations could be explained by the effect of dead ends .

\section{References}

[1] Wu Kai, Xie Hengkun and Ge Jingpang, percolation and Dielectric Breakdown,Electrical Engineering Department of Xi'an Jiaotong University, Xi’an. 710049, P.R.China,1994.

[2] Nir Tessler, Yevgeni Preezant, Noam Rappaport, and Yohai Roichman, «Charge Transport in Disordered Organic Materials and Its Relevance to Thin-Film Devices: A Tutorial Review», Adv. Mater. 2009, 21, pp2741-2761. http://dx.doi.org/10.1002/adma.200803541

[3] D.I.Aladashvili., Z.A.Adamiya., K.G.Lavadovskii., E.Levin., B.I.Shkloskii., «Nonohmic hopping conductivity of weakly compensated semiconductors », Sov Phys semicond 24 (2),234-249 Fev1990

[4] B.Hadri., «Etude de la conductivité électrique en régime non linéaire dans les matériaux inhomogènes », Thèse de Magister (1994), p57, Université d'Oran Es-Sénia, Algérie.

[5] B. Hadri, J. Martinez-Vega, «Investigation of a negative differential conductance in insulating polymers for high electric fields by percolation approach », RS-RIGE Volume 11, pp410,419 